\title{
Preparation and Characterization of Nano-Tetracalcium Phosphate Coating on Titanium Substrate
}

\author{
A.M.Fathi ${ }^{1, *}$, H.K. Abd El-Hamid ${ }^{2}$, M.M. Radwan ${ }^{2}$ \\ ${ }^{1}$ Physical Chemistry Department, National Research Centre, Dokki, Giza12622, Egypt \\ ${ }^{2}$ Ceramics Department, National ResearchCentre,Dokki, Giza 12622, Egypt \\ *E-mail: amfathi70@yahoo.com
}

doi: $10.20964 / 110403164$

Received: 7 December 2015 / Accepted: 28 January 2015 / Published: 1 March 2016

\begin{abstract}
Nano-tetracalcium phosphate TTCP was prepared by solid-state reaction at high temperature and characterized by X-ray diffraction (XRD), Infrared spectroscopy (IR), and Transmission Electron Microscope (TEM). The results show the formation of fine particles size represent agglomerates of very small spherical well-ordered nanoparticles of TTCP with dimensions range from 10-17.8 nm. An inexpensive and relatively simple electrochemical method was used to promote the nucleation of TTCP on Titanium metal and assist the deposition of TTCP from slightly supersaturated solution. The microstructure of the deposited $\mathrm{Ca}-\mathrm{P}-\mathrm{O}$ was investigated by scanning electron microscope (SEM) and electron dispersive x-ray (EDX). A thin film of Ca-P-O deposit was observed after 30min of electrodeposition of TTCP at $-1.7 \mathrm{~V}$ and $3 \mathrm{hrs}$ immersion in the same solution at ambient temperature and neutral slightly supersaturated TTCP solution with $\mathrm{Ca} / \mathrm{P}$ ratio of $\approx 2$. The growth of scarce crystals on this layer composed of primary agglomerated particles with large size after 3days immersion in a simulated body fluid containing phosphate (SBF2) with $\mathrm{Ca} / \mathrm{P}$ ratio of 1.59 . The results indicated that, the TTCP deposited first at Ti surface and it hydrolyzed to hydroxyapatite after few days of immersion in SBF2. Using this inexpensive coating protocol, the TTCP/Ti surface which deposited at different cathodic potentials shows a significant corrosion protection in the two synthesized body fluid solutions when compared with undeposited Ti. The corrosion current density decreases with the increase of immersion time.
\end{abstract}

Keywords: Titanium, Tetracalcium phosphate, Hydroxyapatite, Biocements, Electrodeposition, Corrosion.

\section{$\underline{\text { FULL TEXT }}$}

(C) 2016 The Authors. Published by ESG (www.electrochemsci.org). This article is an open access article distributed under the terms and conditions of the Creative Commons Attribution license (http://creativecommons.org/licenses/by/4.0/). 